\title{
International publications of authors from Bosnia and Herzegovina in Current Contents indexed publications in the first half of $2017^{\star}$
}

Alibegovic E, Kurtcehajic A, Hujdurovic A, Zeco A, Kurtcehajic D. Acute submucosal hematoma: an adverse event of routine colonoscopy. Gastrointest Endosc. 2017 Aug;86(2):409-411. doi: 10.1016/j. gie.2017.02.009. Epub 2017 Feb 24.

Alić A, Hodžić A, Škapur V, Alić AŠ, Prašović S, Duscher GG. Fatal pulmonary cysticercosis caused by Cysticercus longicollis in a captive ring-tailed lemur (Lemur catta). Vet Parasitol. 2017 Jul 15;241:1-4. doi: 10.1016/j.vetpar.2017.05.004. Epub 2017 May 12.

Arslan A. Imaging genetics of schizophrenia in the post-GWAS era. Prog Neuropsychopharmacol Biol Psychiatry. 2018 Jan 3;80(Pt B):155-165. doi: 10.1016/j.pnpbp.2017.06.018. Epub 2017 Jun 20.

Asic A, Kurtovic-Kozaric A, Besic L, Mehinovic L, Hasic A, Kozaric M, Hukic M, Marjanovic D. Chemical toxicity and radioactivity of depleted uranium: The evidence from in vivo and in vitro studies. Environ Res. 2017 Jul;156:665-673. doi: 10.1016/j.envres.2017.04.032. Epub 2017 May 12.

Babić D, Vasilj I, Mimica M. The Faculty of Health Studies at the University of Mostar in the Service of Health. Psychiatr Danub. 2017 May;29(Suppl 2):96-100.

Besic L, Muhovic I, Asic A, Kurtovic-Kozaric A. Metaanalysis of depleted uranium levels in the Balkan region. J Environ Radioact. 2017 Jun;172:207-217. doi: 10.1016/j.jenvrad.2017.03.013. Epub 2017 Apr 7.

Budinski-Petković L, Lončarević I, Dujak D, Karač A, Šćepanović JR, Jakšić ZM, Vrhovac SB. Particle morphology effects in random sequential adsorption. Phys Rev E. 2017 Feb;95(2-1):022114. doi: 10.1103/ PhysRevE.95.022114. Epub 2017 Feb 13.

Catic A, Kurtovic-Kozaric A, Johnson SH, Vasmatzis G, Pins MR, Kogan J. A novel cytogenetic and molecular characterization of renal metanephric adenoma: Identification of partner genes involved in translocation $\mathrm{t}(9 ; 15)(\mathrm{p} 24 ; \mathrm{q} 24)$. Cancer Genet. 2017 Aug;214-215:9-15. doi: 10.1016/j. cancergen.2017.03.001. Epub 2017 Mar 16.

Ceci S, Hadžimehmedović M, Osmanović H, Percan A, Zauner B. Fundamental properties of resonances. Sci Rep. 2017 Mar 27;7:45246. doi: 10.1038/ srep45246.

Cubro H, Kashani K, Gajic O. Learning Health Care System: Pragmatic Comparison of Crystalloid Choice in a Medical Intensive Care Unit. Am J Respir Crit Care Med. 2017 May 15;195(10):1287-1289. doi: 10.1164/rccm.201610-2093ED.

Cvitković I. From conflict and misunderstanding to respect. Psychiatr Danub. 2017 Apr;29 Suppl 1:58-63.

Čakar J, Pilav A, Džehverović M, Ahatović A, Haverić S, Ramić J, Marjanović D. DNA Identification of Commingled Human Remains from the Cemetery Relocated by Flooding in Central Bosnia and Herzegovina. J Forensic Sci. 2017 May 11. doi: 10.1111/1556-4029.13535. [Epub ahead of print]

Dervisevic M, Dervisevic E, Senel M, Cevik E, Yildiz HB, Camurlu P. Construction of ferrocene modified conducting polymer based amperometric urea biosensor. Enzyme Microb Technol. 2017 Jul;102:5359. doi: 10.1016/j.enzmictec.2017.04.002. Epub 2017 Apr 7.

Di Donato I, Bianchi S, De Stefano N, Dichgans M, Dotti MT, Duering M, Jouvent E, Korczyn AD, Lesnik-Oberstein SA, Malandrini A, Markus HS, Pantoni L, Penco S, Rufa A, Sinanović O, Stojanov D, Federico A. Cerebral Autosomal Dominant Arteriopathy with Subcortical Infarcts and Leukoencephalopathy (CADASIL) as a model of small vessel disease: update on clinical, diagnostic, and management aspects. BMC Med. 2017 Feb 24;15(1):41. doi: 10.1186/s12916-017-0778-8.

${ }^{\star}$ Data for this survey were collected from PubMed/MEDLINE using the keywords Bosnia and Herzegovina and 2017. The search was performed on the $8^{\text {th }}$ of November 2017. 
Dujic T, Zhou K, Donnelly LA, Leese G, Palmer CNA, Pearson ER. Interaction between variants in the CYP2C9 and POR genes and the risk of sulfonylurea-induced hypoglycaemia: A GoDARTS Study. Diabetes Obes Metab. 2017 Jun 28. doi: 10.1111/dom.13046. [Epub ahead of print]

Dujic T, Zhou K, Yee SW, van Leeuwen N, de Keyser CE, Javorský M, Goswami S, Zaharenko L, Hougaard Christensen MM, Out M, Tavendale R, Kubo M, Hedderson MM, van der Heijden AA, Klimčáková L, Pirags V, Kooy A, Brøsen K, Klovins J, Semiz S, Tkáč I, Stricker BH, Palmer C, ‘t Hart LM, Giacomini KM, Pearson ER. Variants in Pharmacokinetic Transporters and Glycemic Response to Metformin: A Metgen Meta-Analysis. Clin Pharmacol Ther. 2017 Jun;101(6):763-772. doi: 10.1002/cpt.567. Epub 2017 Feb 3.

Duraković E. Taking the Text out of Context as One of the Causes of the Crisis in the Contemporary Muslim World. Psychiatr Danub. 2017 Apr;29 Suppl 1:73-78.

Duric NS, Assmus J, Gundersen D, Duric Golos A, Elgen IB. Multimodal treatment in children and adolescents with attention-deficit/ hyperactivity disorder: a 6-month follow-up. Nord J Psychiatry. 2017 Jul;71(5):386-394. doi: 10.1080/08039488.2017.1305446. Epub 2017 Mar 27.

Erdem H, Inan A, Guven E, Hargreaves S, Larsen L, Shehata G, Pernicova E, Khan E, Bastakova L, Namani S, Harxhi A, Roganovic T, Lakatos B, Uysal S, Sipahi OR, Crisan A, Miftode E, Stebel R, Jegorovic B, Fehér Z, Jekkel C, Pandak N, Moravveji A, Yilmaz H, Khalifa A, Musabak U, Yilmaz S, Jouhar A, Oztoprak N, Argemi X, Baldeyrou M, Bellaud G, Moroti RV, Hasbun R, Salazar L, Tekin R, Canestri A, Čalkić L, Praticò L, Yilmaz-Karadag F, Santos L, Pinto A, Kaptan F, Bossi P, Aron J, Duissenova A, Shopayeva G, Utaganov B, Grgic S, Ersoz G, Wu AK, Lung KC, Bruzsa A, Radic LB, Kahraman H, MomenHeravi M, Kulzhanova S, Rigo F, Konkayeva M, Smagulova Z, Tang T, Chan P, Ahmetagic S, PorobicJahic H, Moradi F, Kaya S, Cag Y, Bohr A, Artuk C, Celik I, Amsilli M, Gul HC, Cascio A, Lanzafame M, Nassar M. The burden and epidemiology of community-acquired central nervous system infections: a multinational study. Eur J Clin Microbiol Infect Dis. 2017 Apr 10. doi: 10.1007/s10096-0172973-0. [Epub ahead of print]

Fetić B, Milošević DB. Numerical solution of the time-dependent Schrödinger equation for $\mathrm{H}_{-}\{2\} \wedge\{+\}$ ion with application to high-harmonic generation and above-threshold ionization. Phys
Rev E. 2017 May;95(5-1):053309. doi: 10.1103/ PhysRevE.95.053309. Epub 2017 May 31.

Fidahic M, Jelicic Kadic A, Radic M, Puljak L. Celecoxib for rheumatoid arthritis. Cochrane Database Syst Rev. 2017 Jun 9;6:CD012095. doi: 10.1002/14651858.CD012095.pub2.

Fontana S, Buttinelli G, Fiore S, Mulaomerovic M, Aćimović J, Amato C, Delogu R, Rezza G, Stefanelli P. Acute flaccid paralysis surveillance in bosnia and herzegovina: Recent isolation of two sabin like type 2 poliovirus. J Med Virol. 2017 Sep;89(9):1678-1681. doi: 10.1002/jmv.24827. Epub 2017 May 23.

Gorički Š, Stanković D, Snoj A, Kuntner M, Jeffery WR, Trontelj P, Pavićević M, Grizelj Z, NăpăruşAljančič M, Aljančič G. Environmental DNA in subterranean biology: range extension and taxonomic implications for Proteus. Sci Rep. 2017 Mar 27;7:45054. doi: 10.1038/srep45054.

Grau-Sánchez J, Foley M, Hlavová R, Muukkonen I, Ojinaga-Alfageme O, Radukic A, Spindler M, Hundevad B. Exploring Musical Activities and Their Relationship to Emotional Well-Being in Elderly People across Europe: A Study Protocol. Front Psychol. 2017 Mar 20;8:330. doi: 10.3389/ fpsyg.2017.00330. eCollection 2017.

Gunarić A, Jurišić K, Šimić D, Penavić JZ, Jozić S, Goluža I. Scabies Mimicing Child Abuse - a Case Report. Psychiatr Danub. 2017 May;29(Suppl 2):145147.

Gutić SJ, Dobrota AS, Leetmaa M, Skorodumova NV, Mentus SV, Pašti IA. Improved catalysts for hydrogen evolution reaction in alkaline solutions through the electrochemical formation of nickelreduced graphene oxide interface. Phys Chem Chem Phys. 2017 May 24;19(20):13281-13293. doi: 10.1039/ c7cp01237c.

Guzvic V, Catic T, Kostic M. Health technology assessment in Central-Eastern and South Europe countries: Bosnia and Herzegovina. Int J Technol Assess Health Care. 2017 Jan;33(3):390-395. doi: 10.1017/S0266462317000058. Epub 2017 Apr 24.

Halilović S. Islamic Civilization in Spain - a Magnificient Example of Interaction and Unity of Religion and Science. Psychiatr Danub. 2017 Apr;29 Suppl 1:64-72.

Hauser G, Blažević I, Salkić N, Poropat G, Giljača V, Bulić Z, Štimac D. Erratum to: Diclofenac sodium versus ceftazidime for preventing pancreatitis after endoscopic retrograde cholangiopancreatography: 
a prospective, randomized, controlled trial. Surg Endosc. 2017 Feb;31(2):611. doi: 10.1007/s00464016-5305-z.

Ibrić N, Ahmetović E, Kravanja Z, Maréchal F, Kermani M. Synthesis of single and interplant non-isothermal water networks. J Environ Manage. 2017 Dec 1;203(Pt 3):1095-1117. doi: 10.1016/j. jenvman.2017.05.001. Epub 2017 Jun 9.

Igić R. Four decades of ocular renin-angiotensin and kallikrein-kinin systems (1977-2017). Exp Eye Res. 2017 May 23;166:74-83. doi: 10.1016/j. exer.2017.05.007. [Epub ahead of print]

Islamagic E, Hasic A, Kurtovic S, Suljovic Hadzimesic E, Mehinovic L, Kozaric M, Kurtovic-Kozaric A. The Efficacy of Generic Imatinib as First- and Second-line Therapy: 3-Year Follow-up of Patients With Chronic Myeloid Leukemia. Clin Lymphoma Myeloma Leuk. 2017 Apr;17(4):238-240. doi: 10.1016/j. clml.2017.02.001. Epub 2017 Feb 16.

Jakšić N, Margetić BA. Comments on the Role of the Character Dimension Self-Transcendence in Suicidal Phenomena. J Clin Psychiatry. 2017 Feb;78(2):e161. doi: 10.4088/JCP.16lr11229.

Jurišić S, Verzak Ž, Jurišić G, Jurić H. Assessment of efficacy of two chlorhexidine mouthrinses on oral hygiene and gingival health in adolescents wearing two types of orthodontic brackets. Int J Dent Hyg. 2017 Jun 28. doi: 10.1111/idh.12299. [Epub ahead of print]

Kandolf Sekulovic L, Peris K, Hauschild A, Stratigos A, Grob JJ, Nathan P, Dummer R, Forsea AM, Hoeller C, Gogas H, Demidov L, Lebbe C, Blank C, Olah J, Bastholt L, Herceg D, Neyns B, Vieira R, Hansson J, Rutkowski P, Krajsova I, BylaiteBucinskiene M, Zalaudek I, Maric-Brozic J, Babovic N, Banjin M, Putnik K, Weinlich G, Todorovic V, Kirov K, Ocvirk J, Zhukavets A, Kukushkina M, De La Cruz Merino L, Ymeri A, Risteski M, Garbe C. More than 5000 patients with metastatic melanoma in Europe per year do not have access to recommended first-line innovative treatments. Eur J Cancer. 2017 Apr;75:313-322. doi: 10.1016/j. ejca.2017.01.012. Epub 2017 Mar 4.

Kasum M, Franulić D, Čehić E, Orešković S, Lila A, Ejubović E. Kisspeptin as a promising oocyte maturation trigger for in vitro fertilisation in humans. Gynecol Endocrinol. 2017 Aug;33(8):583587. doi: 10.1080/09513590.2017.1309019. Epub 2017 Apr 10.
Kővári B, Vranic S, Marchio C, Sapino A, Cserni G. The expression of GHRH and its receptors in breast carcinomas with apocrine differentiation-further evidence of the presence of a GHRH pathway in these tumors. Hum Pathol. 2017 Jun;64:164-170. doi: 10.1016/j.humpath.2017.03.026. Epub 2017 Apr 21.

Krupchanka D, Khalifeh H, Abdulmalik J, ArdilaGómez S, Armiya'u AY, Banjac V, Baranov A, Bezborodovs N, Brecic P, Čavajda Z, de Girolamo G, Denisenko M, Dickens HA, Dujmovic J, Ergovic Novotny D, Fedotov I, Fernández MA, Frankova I, Gasparovic M, Giurgi-Oncu C, Grahovac T, James BO, Jomli R, Kekin I, Knez R, Lanfredi M, Lassman F, Mehta N, Nacef F, Nawka A, Nemirovsky M, Ola BA, Oshodi YO, Ouali U, Peharda T, Razic Pavicic A, Rojnic Kuzman M, Roventa C, Shamenov R, Smirnova D, Smoljanic D, Spikina A, Thornicroft A, Tomicevic M, Vidovic D, Williams P, Yakovleva Y, Zhabenko O, Zhilyaeva T, Zivkovic M, Thornicroft G, Sartorius N. Satisfaction with psychiatric in-patient care as rated by patients at discharge from hospitals in 11 countries. Soc Psychiatry Psychiatr Epidemiol. 2017 Aug;52(8):989-1003. doi: 10.1007/s00127-0171366-0. Epub 2017 Mar 11.

Kurtovic-Kozaric A, Kugic A, Hasic A, Beslija S, Ceric T, Pasic A, Vranic S, Kopric D, Iljazovic E, Todorovic Barbuscia J, Kozaric M, Ibisevic N, Keskic L, Kurtovic S. Long-term outcome of GIST patients treated with delayed imatinib therapy. Eur J Cancer. 2017 Jun;78:118-121. doi: 10.1016/j.ejca.2017.03.024. Epub 2017 Apr 21.

Lansbury LE, Smith S, Beyer W, Karamehic E, Pasic-Juhas E, Sikira H, Mateus A, Oshitani H, Zhao H, Beck CR, Nguyen-Van-Tam JS. Effectiveness of 2009 pandemic influenza $\mathrm{A}(\mathrm{H} 1 \mathrm{~N} 1)$ vaccines: A systematic review and meta-analysis. Vaccine. 2017 Apr 11;35(16):1996-2006. doi: 10.1016/j. vaccine.2017.02.059. Epub 2017 Mar 14.

Liu Y, Friesen JB, Grzelak EM, Fan Q, Tang T, Durić K, Jaki BU, McAlpine JB, Franzblau SG, Chen SN, Pauli GF. Sweet spot matching: A thin-layer chromatography-based countercurrent solvent system selection strategy. J Chromatogr A. 2017 Jun 30;1504:46-54. doi: 10.1016/j.chroma.2017.04.055. Epub 2017 Apr 27.

Ljubojević G, Miljković B, Bućma T, Ćulafić M, Prostran M, Vezmar Kovačević S. Problems, interventions, and their outcomes during the routine work of hospital pharmacists in Bosnia and Herzegovina. Int J Clin Pharm. 2017 Aug;39(4):743749. doi: 10.1007/s11096-017-0491-x. Epub 2017 Jun 9. 
Maraolo AE, Ong DSY, Cortez J, Dedić K, Dušek D, Martin-Quiros A, Maver PJ, Skevaki C, Yusuf E, Poljak M, Sanguinetti M, Tacconelli E; Trainee Association of the European Society of Clinical Microbiology and Infectious Diseases (ESCMID). Personal life and working conditions of trainees and young specialists in clinical microbiology and infectious diseases in Europe: a questionnaire survey. Eur J Clin Microbiol Infect Dis. 2017 Jul;36(7):12871295. doi: 10.1007/s10096-017-2937-4. Epub 2017 Feb 24.

Markotić V, Miljko M, Radančević D, Grle M, Perić I, Arapović AK, Bogdan G. A case report of a long time unrecognized hypochondriac patient wondering through the hospital departments. Psychiatr Danub. 2017 May;29(Suppl 2):142-144.

Martinac M, Babić D, Bevanda M, Vasilj I, Glibo DB, Karlović D, Jakovljević M. Activity of the hypothalamic-pituitary-adrenal axis and inflammatory mediators in major depressive disorder with or without metabolic syndrome. Psychiatr Danub. 2017 Mar;29(1):39-50.

Martinovic V, Vukusic Pusic T, Restovic I, Bocina I, Filipovic N, Saraga-Babic M, Vukojevic K. Expression of Epithelial and Mesenchymal Differentiation Markers in the Early Human Gonadal Development. Anat Rec (Hoboken). 2017 Jul;300(7):1315-1326. doi: 10.1002/ar.23531. Epub 2017 Feb 6.

Michal M, Kazakov DV, Agaimy A, Hosova M, Michalova K, Grossmann P, Steiner P, Skenderi F, Vranic S, Michal M. Whorling cellular perineurioma: A previously undescribed variant closely mimicking monophasic fibrous synovial sarcoma. Ann Diagn Pathol. 2017 Apr;27:74-78. doi: 10.1016/j. anndiagpath.2017.02.001. Epub 2017 Feb 2.

Mijajlović MD, Pavlović A, Brainin M, Heiss WD, Quinn TJ, Ihle-Hansen HB, Hermann DM, Assayag EB, Richard E, Thiel A, Kliper E, Shin YI, Kim YH, Choi S, Jung S, Lee YB, Sinanović O, Levine DA, Schlesinger I, Mead G, Milošević V, Leys D, Hagberg G, Ursin MH, Teuschl Y, Prokopenko S, Mozheyko E, Bezdenezhnykh A, Matz K, Aleksić V, Muresanu D, Korczyn AD, Bornstein NM. Post-stroke dementia - a comprehensive review. BMC Med. 2017 Jan 18;15(1):11. doi: 10.1186/s12916-017-0779-7.

Mirković-Hajdukov M, Spahić TE, Softić R, Bećirović E, Šimić J. Family Atmosphere and Relationships as Predictors of Heroin Addiction. Psychiatr Danub. 2017 May;29(Suppl 2):129-133.

Omanovic-Miklicanin E, Valzacchi S. Development of new chemiluminescence biosensors for determination of biogenic amines in meat. Food Chem. 2017 Nov 15;235:98-103. doi: 10.1016/j. foodchem.2017.05.031. Epub 2017 May 6.

Orlovic M, Tomic V, Vukojevic K, Hudic I, Mandic V, Azinovic I, Soldo D, Kajic M, Soljic V. Decreased expression of MMP-9 in CD8+ cells in placenta with severe preeclampsia. Biotech Histochem. 2017;92(4):288-296. doi: 10.1080/10520295.2017.1309069. Epub 2017 May 12.

Ostojic M, Soljic V, Vukojevic K, Dapic T. Immunohistochemical characterization of early and advanced knee osteoarthritis by NF-kB and iNOS expression. J Orthop Res. 2017 Sep;35(9):1990-1997. doi: 10.1002/jor.23504. Epub 2017 May 3.

Pehar M, Sisic N, Sekulic D, Coh M, Uljevic O, Spasic M, Krolo A, Idrizovic K. Analyzing the relationship between anthropometric and motor indices with basketball specific pre-planned and non-planned agility performances. J Sports Med Phys Fitness. 2017 May 9. doi: 10.23736/S0022-4707.17.07346-7. [Epub ahead of print]

Pilav A, Pojskić N, Ahatović A, Džehverović M, Čakar J, Marjanović D. Allele frequencies of 15 STR loci in Bosnian and Herzegovinian population. Croat Med J. 2017 Jun 14;58(3):250-256.

Postolache D, Popescu F, Paule L, Ballian D, Zhelev P, Fărcaş S, Paule J, Badea O. Unique postglacial evolution of the hornbeam (Carpinus betulus L.) in the Carpathians and the Balkan Peninsula revealed by chloroplast DNA. Sci Total Environ. 2017 Dec 1;599600:1493-1502. doi: 10.1016/j.scitotenv.2017.05.062. Epub 2017 May 17.

Puljak L, Marin A, Vrdoljak D, Markotic F, Utrobicic A, Tugwell P. Celecoxib for osteoarthritis. Cochrane Database Syst Rev. 2017 May 22;5:CD009865. doi: 10.1002/14651858.CD009865.pub2.

Rastović P, Trninić Z, Galić G, Brekalo Z, Lesko J, Pavlović M. Accuracy of Modified Alvarado Score, Eskelinen Score and Ohmann Score in Diagnosing Acute Appendicitis. Psychiatr Danub. 2017 May;29(Suppl 2):134-141.

Ravlija J, Vasilj I, Babic D, Marijanovic I. Public Health Achievements and Challenges: Symposium of the University of Mostar Faculty of Health Studies. Psychiatr Danub. 2017 May;29(Suppl 2):101-103.

Skelin M, Lucijanić T, Amidžić Klarić D, Rešić A, Bakula M, Liberati-Čizmek AM, Gharib H, Rahelić D. Factors Affecting Gastrointestinal Absorption of Levothyroxine: A Review. Clin 
Ther. 2017 Feb;39(2):378-403. doi: 10.1016/j. clinthera.2017.01.005. Epub 2017 Jan 30.

Skenderi F, Ulamec M, Vanecek T, Martinek P, Alaghehbandan R, Foix MP, Babankova I, Montiel DP, Alvarado-Cabrero I, Svajdler M, Dubinský P, Cempirkova D, Pavlovsky M, Vranic S, Daum $\mathrm{O}$, Ondic O, Pivovarcikova K, Michalova K, Hora M, Rotterova P, Stehlikova A, Dusek M, Michal M, Hes O. Warthin-like papillary renal cell carcinoma: Clinicopathologic, morphologic, immunohistochemical and molecular genetic analysis of 11 cases. Ann Diagn Pathol. 2017 Apr;27:48-56. doi: 10.1016/j.anndiagpath.2017.01.005. Epub 2017 Jan 19.

Soljic M, Mrklic I, Tomic S, Omrcen T, Sutalo N, Bevanda M, Vrdoljak E. Prognostic value of vitamin $\mathrm{D}$ receptor and insulin-like growth factor receptor 1 expression in triple-negative breast cancer. J Clin Pathol. 2017 Jun 29. pii: jclinpath-2016-204222. doi: 10.1136/jclinpath-2016-204222. [Epub ahead of print]

Streit S, Verschoor M, Rodondi N, Bonfim D, Burman RA, Collins C, Biljana GK, Gintere S, Gómez Bravo R, Hoffmann K, Iftode C, Johansen KL, Kerse N, Koskela TH, Peštić SK, Kurpas D, Mallen CD, Maisoneuve H, Merlo C, Mueller Y, Muth C, Šter MP, Petrazzuoli F, Rosemann T, Sattler M, Švadlenková Z, Tatsioni A, Thulesius H, Tkachenko V, Torzsa P, Tsopra R, Canan T, Viegas RPA, Vinker S, de Waal MWM, Zeller A, Gussekloo J, Poortvliet RKE. Variation in GP decisions on antihypertensive treatment in oldest-old and frail individuals across 29 countries. BMC Geriatr. 2017 Apr 20;17(1):93. doi: 10.1186/s12877-017-0486-4.

Suručić R, Kundaković T, Lakušić B, Drakul D, Milovanović SR, Kovačević N. Variations in Chemical Composition, Vasorelaxant and Angiotensin I-Converting Enzyme Inhibitory Activities of Essential Oil from Aerial Parts of Seseli pallasii Besser (Apiaceae). Chem Biodivers. 2017 May;14(5). doi: 10.1002/cbdv.201600407. Epub 2017 Apr 17.

Svirčev Z, Drobac D, Tokodi N, Mijović B, Codd GA, Meriluoto J. Toxicology of microcystins with reference to cases of human intoxications and epidemiological investigations of exposures to cyanobacteria and cyanotoxins. Arch Toxicol. 2017 Feb;91(2):621-650. doi: 10.1007/s00204-016-1921-6. Epub 2017 Jan 2.

Šimić D, Penavić JZ, Babić D, Gunarić A. Psychological Status and Quality of Life in Acne Patients Treated with Oral Isotretinoin. Psychiatr Danub. 2017 May;29(Suppl 2):104-110.
Šimunović M, Tokmakčija S, Pavlović M, Babić R, Vasilj M, Martinac M, Vasilj I, Babić D. The Impact of Religiosity on Quality of Life and Psychological Symptoms in Chronic Mental Patients. Psychiatr Danub. 2017 May;29(Suppl 2):118-123.

Škrbić R, Stojiljković MP, Ćetković SS, Dobrić S, Jeremić D, Vulović M. Naloxone Antagonizes Somaninduced Central Respiratory Depression in Rats. Basic Clin Pharmacol Toxicol. 2017 Jun;120(6):615620. doi: 10.1111/bcpt.12745. Epub 2017 Mar 15.

Špirić Z, Erić M, Eri Ž. Lymphatic invasion and the Shields index in predicting melanoma metastases. J Plast Reconstr Aesthet Surg. 2017 Nov;70(11):16461652. doi: 10.1016/j.bjps.2017.05.056. Epub 2017 Jun 13.

Taler-Verčič A, Hasanbašić S, Berbić S, Stoka V, Turk D, Žerovnik E. Proline Residues as Switches in Conformational Changes Leading to Amyloid Fibril Formation. Int J Mol Sci. 2017 Mar 7;18(3). pii: E549. doi: 10.3390/ijms18030549.

Templ B, Templ M, Filzmoser P, Lehoczky A, Bakšienè E, Fleck S, Gregow H, Hodzic S, Kalvane G, Kubin E, Palm V, Romanovskaja D, Vuc vetic V, Žust A, Czúcz B; NS-Pheno Team. Phenological patterns of flowering across biogeographical regions of Europe. Int J Biometeorol. 2017 Jul;61(7):1347-1358. doi: 10.1007/s00484-017-1312-6. Epub 2017 Feb 20.

Udovicic-Gagula D, Ahmovic A, Bilalovic N, Doric M. Expression of Ki-67 and Estrogen Receptor Beta in Primary Cutaneous Melanoma as a Potential Indicator of Regional Lymph Node Positivity. Appl Immunohistochem Mol Morphol. 2017 May 25. doi: 10.1097/PAI.0000000000000530. [Epub ahead of print]

Välitalo PA, Kemppainen H, Kulo A, Smits A, van Calsteren K, Olkkola KT, de Hoon J, Knibbe CAJ, Allegaert K. Body weight, gender and pregnancy affect enantiomer-specific ketorolac pharmacokinetics. Br J Clin Pharmacol. 2017 Sep;83(9):1966-1975. doi: 10.1111/bcp.13311. Epub 2017 May 14.

Vasilj M, Klarić M, Vrkić N, Mikulić I, Boras MM, Jelić-Knezović N, Šoljić V. Kappa Free Light Chains in Cerebrospinal Fluid of Patients with Identified Oligoclonal Immunoglobulin G. Psychiatr Danub. 2017 May;29(Suppl 2):124-128.

Vukojević M, Cvitković T, Splavski B, Ostojić Z, Šumanović-Glamuzina D, Šimić J. Prevalence of Intellectual Disabilities and Epilepsy in Different 
Forms of Spastic Cerebral Palsy in Adults. Psychiatr Danub. 2017 May;29(Suppl 2):111-117.

Vukojević Z, Ilić TV, Dominović-Kovačević A, Grgić S, Mavija S. Neurosarcoidosis and multiple intracerebral hematomas: An unusual clinical presentation. J Neurol Sci. 2017 Aug 15;379:22-24. doi: 10.1016/j.jns.2017.05.030. Epub 2017 May 17.

Winkler P, Krupchanka D, Roberts T, Kondratova L, Machů V, Höschl C, Sartorius N, Van Voren R, Aizberg O, Bitter I, Cerga-Pashoja A, Deljkovic A, Fanaj N, Germanavicius A, Hinkov H, Hovsepyan A, Ismayilov FN, Ivezic SS, Jarema M, Jordanova V, Kukić S, Makhashvili N, Šarotar BN, Plevachuk O, Smirnova D, Voinescu BI, Vrublevska J, Thornicroft G. A blind spot on the global mental health map: a scoping review of 25 years' development of mental health care for people with severe mental illnesses in central and eastern Europe. Lancet Psychiatry. 2017 Aug;4(8):634-642. doi: 10.1016/S22150366(17)30135-9. Epub 2017 May 8.
Zenic N, Ban D, Jurisic S, Cubela M, Rodek J, Ostojic L, Jelicic M, Bianco A, Sekulic D. Prospective Analysis of the Influence of Sport and Educational Factors on the Prevalence and Initiation of Smoking in Older Adolescents from Croatia. Int J Environ Res Public Health. 2017 Apr 20;14(4). pii: E446. doi: 10.3390/ijerph14040446.

Zerem E, Kunosić S, Handanagić A, Jahić D, Zerem D, Zerem O. Minimally Invasive Treatment for Appendiceal Mass Formed After Acute Perforated Appendicitis. Surg Laparosc Endosc Percutan Tech. 2017 Jun;27(3):132-138. doi: 10.1097/ SLE.0000000000000404.

Zukic S, Sinanovic O, Zonic L. Two year outcomes of poststroke writing and reading disorders. Appl Neuropsychol Adult. 2017 May 10:1-5. doi: 10.1080/23279095.2017.1320285. [Epub ahead of print]

by Nerma Tanović 